\title{
Resource Allocation for OCI Reduction in OFDM- based Asynchronous Cellular Systems
}

\author{
Jin-Woo Lee and Yong-Hwan Lee \\ School of Electrical Engineering and INMC \\ Seoul National University \\ Kwanak P. O. Box 34, Seoul 151-600 Korea \\ e-mail: jinu@ttl.snu.ac.kr, ylee@snu.ac.kr
}

\begin{abstract}
Orthogonal frequency division multiplexing (OFDM) is considered as one of the most promising techniques for next generation mobile systems. When the OFDM is applied to cellular environments, it may suffer from so-called other-cell interference (OCI). In this paper, we propose a novel resource allocation scheme in OFDM-based asynchronous cellular systems. It can reduce the OCI by exploiting the repetitive property of a cyclic prefix (CP) of OFDM symbols. The proposed scheme can be applied to OFDM-frequency division multiple access (FDMA) and OFDM-code division multiple access (CDMA) systems. Computer simulation results show that the proposed scheme can reduce the OCI by nearly up to $1 \mathrm{~dB}$ in a typical cellular OFDM environment, compared to the conventional scheme.
\end{abstract}

\section{INTRODUCTION}

Broadband wireless packet access systems have attracted for the achievement of high-speed transmission capacity. Orthogonal frequency division multiplexing (OFDM) is a very promising scheme for this purpose due to the simplicity of channel equalization in severely frequency selective wireless channel [1]-[3]. However, it may suffer from other-cell interference (OCI) when it is applied to cellular environments that use a single frequency band for all cells [4].

Most of previous studies have considered the use of interference averaging and/or avoidance techniques to mitigate the OCI. OCI averaging schemes require a simple transceiver structure and can easily control the radio resource by using spread spectrum and/or frequency hopping $(\mathrm{FH})$ techniques. Typical OCI averaging schemes consider the use of OFDMcode division multiplexing (CDM) and FH-OFDM [5], [6]. The OCI averaging schemes can provide link-level diversity as a result of channel averaging effect. However, they may not provide significant performance improvement because they cannot sufficiently reduce the amount of OCI. On the other hand, OCI avoidance schemes can reduce the interference by dynamically preventing adjacent base stations (BSs) from using the same frequency resources as the incumbent BS uses. Dynamic packet access (DPA) and fractional frequency reuse (FFR) are typical OCI avoidance schemes [7], [8]. However, conventional OCI avoidance schemes may not be applicable to

This work was in part supported by the Ministry of Information \& Communications, Korea, under the Information Technology Research Center (ITRC) Support Program.
OFDM-based asynchronous cellular systems due to its inherited timing difference between the BSs [9].

In this paper, we consider a novel resource allocation scheme that can reduce the OCI in OFDM-based asynchronous cellular systems. By reducing the power of the last portion of the OFDM symbol corresponding to the cyclic prefix (CP), the proposed resource allocation scheme can noticeably reduce the OCI. The proposed scheme can easily be applied to OFDMFDMA and OFDM-CDMA-based cellular systems.

Following Introduction, the proposed OCI reducing scheme is described in Section II. The proposed resource allocation method is applied to OFDM-based cellular systems in Section III. The performance of the proposed schemes is verified by computer simulation in Section IV. Finally, conclusions are summarized in Section V.

\section{Proposed OCI Reduction Scheme}

The OFDM system transmits the signal by splitting broadband spectrum into a number of narrowband subchannels to mitigate frequency selective fading. Since it can be assumed that the transmitted signal in each subchannel undergoes flat fading, the receiver can recover the signal using a simple onetap equalizer. To mitigate the inter-symbol interference (ISI) and inter-carrier interference (ICI) due to multi-path delay spread, the OFDM transmitter inserts a $\mathrm{CP}$ at the beginning of each OFDM symbol [1]. Since the CP itself is a redundancy requiring additional power, it may be desirable to reduce the power of the CP. Then, the average transmit power can be reduced, reducing the power of OCI at the receiver in an OFDM-based asynchronous cellular system.

In a conventional OFDM system, the $\mathrm{CP}$ is generated as a replica of the last portion of the OFDM symbol with the same power and thus the average transmit power is unchanged during the OFDM symbol interval as illustrated in Fig. 1 (a). To reduce the power of the $\mathrm{CP}$, it is required to design the OFDM symbol to have low power in its last portion corresponding to the CP. Fig. 1 (b) illustrates the design of OFDM symbols for the proposed scheme, where $N$ is the OFDM symbol duration (i.e., the number of subcarriers in the frequency domain) and $N_{g}$ is the CP duration in the sample time domain. 


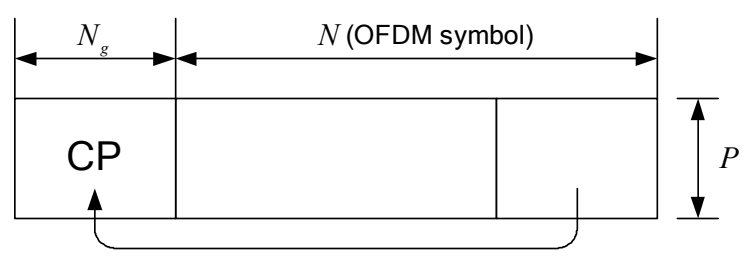

(a) Conventional OFDM signal

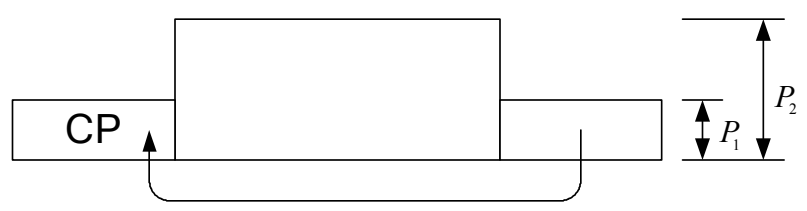

(b) Proposed OFDM signal

Fig. 1. The concept of signal power reduction.

As illustrated in Fig. 1, let $P_{1}$ and $P_{2}$ be the average power of the last portion of the OFDM symbol corresponding to the $\mathrm{CP}$ and the rest of the OFDM symbol, respectively. Thus, the average signal power can be represented as

$$
P=\frac{N_{g} P_{1}+\left(N-N_{g}\right) P_{2}}{N} .
$$

Fig. 2 illustrates the signal distribution when the proposed signaling is applied to an asynchronous OFDM cellular system. Since the signals from the intra BS are synchronized to the receiver, the power reduction of the last portion of the OFDM symbol corresponding to the $\mathrm{CP}$ does not affect the reception performance. However, it can be seen that the average OCI power from the adjacent BSs (or other cells) is reduced in the presence of symbol timing misalignment among the transceivers in this asynchronous cellular system ${ }^{1}$.

The OCI power in Fig. 2 can be represented as

$$
P^{\prime}(\varepsilon)= \begin{cases}\frac{N_{g} P_{1}+\left(N-N_{g}\right) P_{2}}{N}, & 0 \leq \varepsilon<N_{g} \\ \frac{\varepsilon P_{1}+(N-\varepsilon) P_{2}}{N}, & N_{g} \leq \varepsilon<2 N_{g} \\ \frac{2 N_{g} P_{1}+\left(N-2 N_{g}\right) P_{2}}{N}, & 2 N_{g} \leq \varepsilon<N \\ \frac{\left(N+2 N_{g}-\varepsilon\right) P_{1}+\left(\varepsilon-2 N_{g}\right) P_{2}}{N}, & N \leq \varepsilon<N_{g}+N\end{cases}
$$

where $\varepsilon$ denotes the timing offset between the two transceivers. If we assume that $\varepsilon$ is uniformly distributed ${ }^{2}$,

\footnotetext{
${ }^{1}$ In an OFDM-based synchronous cellular system, on the other hand, the $\mathrm{OCI}$ reduction gain cannot be achieved since the power reduced $\mathrm{CP}$ of OCI at the outside of the DFT window is also perfectly removed as that of signal from the intra BS (i.e., $P=P^{\prime}(\varepsilon)$ when $0 \leq \varepsilon<N_{g}$ ).

${ }^{2}$ Since $\varepsilon$ is slowly varying due to the propagation delay between the two transceivers, this assumption is practically reasonable.
}

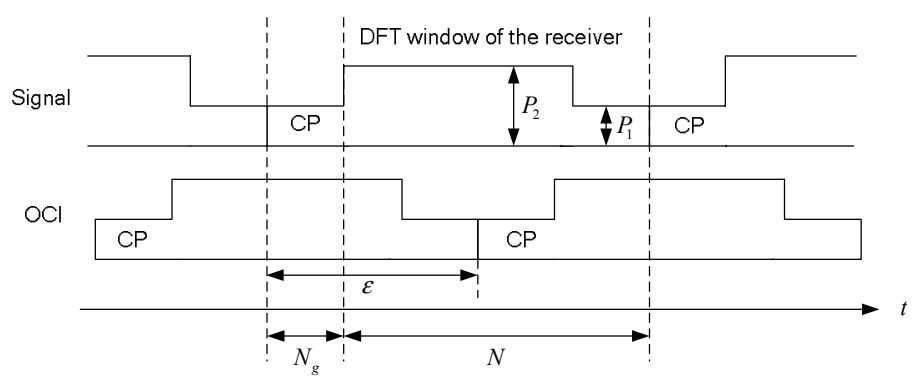

Fig. 2. OCI power reduction in OFDM-based asynchronous cellular systems.

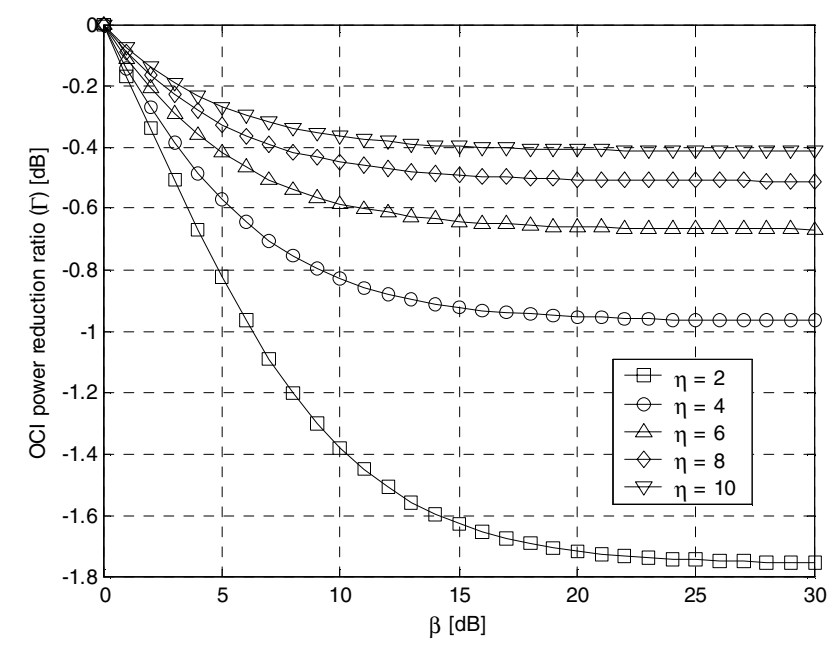

Fig. 3. The average OCI power reduction ratio as a function of $\eta$ and $\beta$.

the average power of the OCI is changed by the proposed scheme as

$$
\begin{aligned}
P^{\prime} & =E\left[P^{\prime}(\varepsilon)\right] \\
& =\frac{2 N_{g} P_{1}+\left(N-N_{g}\right) P_{2}}{N+N_{g}}
\end{aligned}
$$

where $E[X]$ denotes the expectation of $X$. Note that the average power of the OCI in the conventional scheme is $P$. Letting $\eta=N / N_{g}$ and $\beta=P_{2} / P_{1}$, define the OCI power reduction ratio as

$$
\begin{aligned}
\Gamma & =\frac{P^{\prime}}{P} \\
& =\frac{\eta}{\eta+1}\left(1+\frac{1}{1+(\eta-1) \beta}\right) .
\end{aligned}
$$

Fig. 3 depicts the amount of OCI power reduction according to the values of $\eta$ and $\beta$. It can be seen that the gain of the proposed scheme over the conventional one increases as $\eta$ 


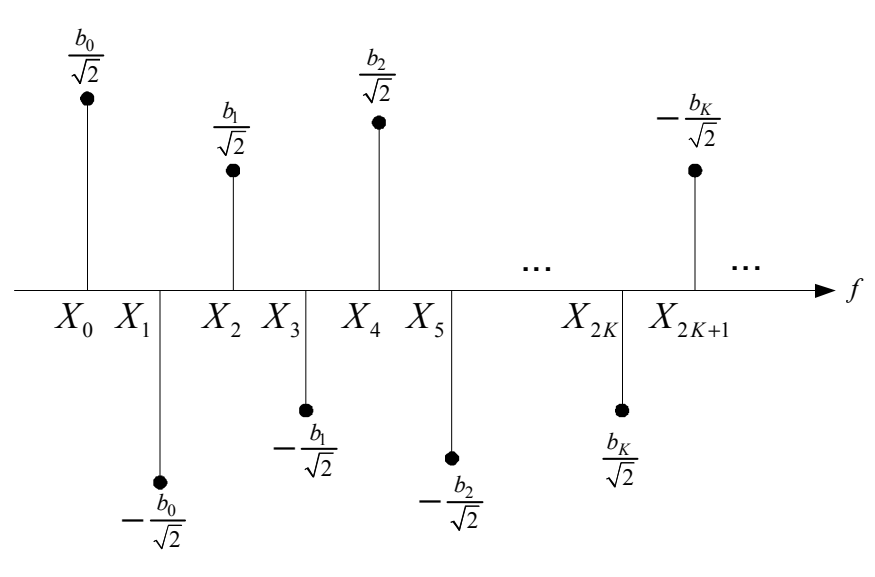

Fig. 4. The proposed resource allocation scheme.

decreases and/or $\beta$ increases. Since $\eta$ is a fixed parameter in practice $^{3}$, the performance can be improved by increasing $\beta$.

To increase $\beta$, we propose a novel resource allocation method in multi-user environments. The proposed resource scheme allocates each pair of data symbols to the adjacent subcarriers with opposite signs as

$$
X_{n}= \begin{cases}b_{n / 2} / \sqrt{2}, & n=0,2, \ldots, N-2 \\ -b_{(n-1) / 2} / \sqrt{2}, & n=1,3, \ldots, N-1\end{cases}
$$

where $b_{k}$ is the data symbol of user $k$ and $X_{n}$ is the transmit signal at the $n$-th subcarrier in the frequency domain. Fig. 4 illustrates a design example when the number of users $K$ is less than $N / 2$, where $N$ is the number of subcarriers. This proposed resource allocation method yields the OFDM signal to have a $\cap$-shaped power characteristic in the time domain (refer to Appendix). Thus, the proposed scheme can increase $\beta$ compared to the conventional scheme, reducing the average OCI power without the increase of complexity.

\section{RESOURCE ALLOCATION FOR MULTI-USER OFDM SYSTEMS}

Unless all the resources (e.g., subcarriers in the OFDMFDMA and spreading codes in the OFDM-CDMA) of multiuser OFDM systems are fully utilized for the signal transmission (i.e., no room for the signal design with increased $\beta$ ), we can reduce the power of the $\mathrm{CP}$ by exploiting the proposed resource allocation scheme described in Section II. We consider the resource allocation for OFDM-FDMA and OFDM-CDMA systems.

\section{A. OFDM-FDMA System}

\footnotetext{
${ }^{3}$ In practice, $\eta$ is designed by considering the maximum delay and Doppler spread according to the system requirements [10].
}

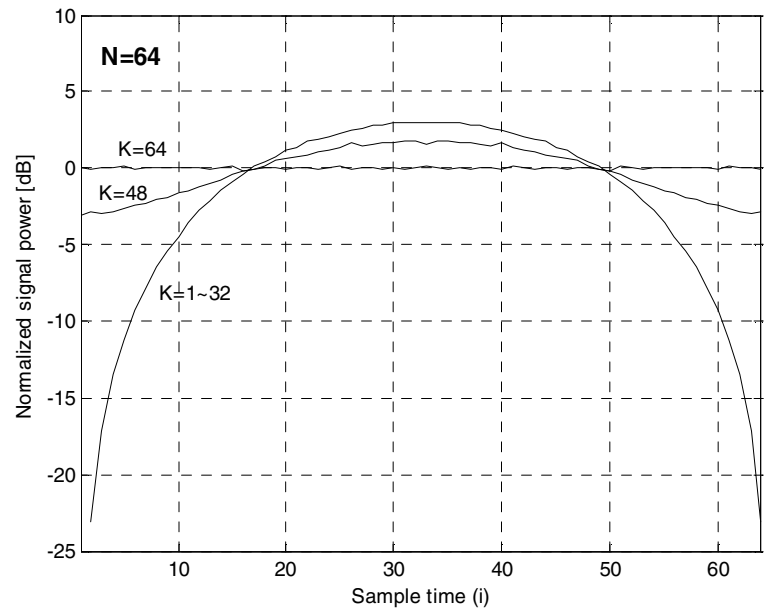

Fig. 5. Power of the proposed OFDM-FDMA signal when $N=64$.

The OFDM-FDMA system transmits multi-user signals by allocating each user signal to different subcarriers. Let $m_{F}(n)$ be the symbol allocated to the $n$-th subcarrier. Assuming that $b_{k}$ is zero for $k>K$, the proposed scheme can allocate the resource for the OFDM-FDMA signal as

$$
\left\{\begin{array}{l}
m_{F}(2 n)=\left(b_{n}+b_{N / 2+n}\right) / \sqrt{2} \\
m_{F}(2 n+1)=\left(-b_{n}+b_{N / 2+n}\right) / \sqrt{2}
\end{array}\right.
$$

where $n=0, \ldots, N / 2-1$. When $K \leq N / 2$, each pair of symbols allocated to the adjacent subcarriers will have opposite signs as (5). When $K>N / 2,(K-N / 2)$ pairs of symbols allocated to the adjacent subcarries do not have opposite signs. This allocation scheme can easily be applicable to an OFDMFDMA system since it only requires a simple linear processor to demodulate the data symbol. Fig. 5 depicts the average signal power of the OFDM symbol for different values of $K$ when $N=64$. It can be seen that the OFDM signal has a $\cap$ shaped power characteristic when $K<N$ and the average signal power corresponding to the $\mathrm{CP}$ portion is noticeably reduced when $K \leq N / 2$.

\section{B. OFDM-CDMA System}

The OFDM-CDMA system transmits multi-user signals by using orthogonal spreading codes. Real-valued binary codes (e.g., Walsh-Hardamard (WH) codes) are often employed as the spreading code due to their simplicity [11]. If the spreading factor $L$ is equal to $N$, there can exist $N$ spreading codes. The optimum resource allocation rule for the reduction of $\beta$ can be obtained by exhaustive search using the spectral properties of the WH code [12]. However, since the optimum allocation rule is associated with the values of $N$ and $\eta$, it may be desirable to employ a suboptimum allocation rule robust to the variation of these parameters. 


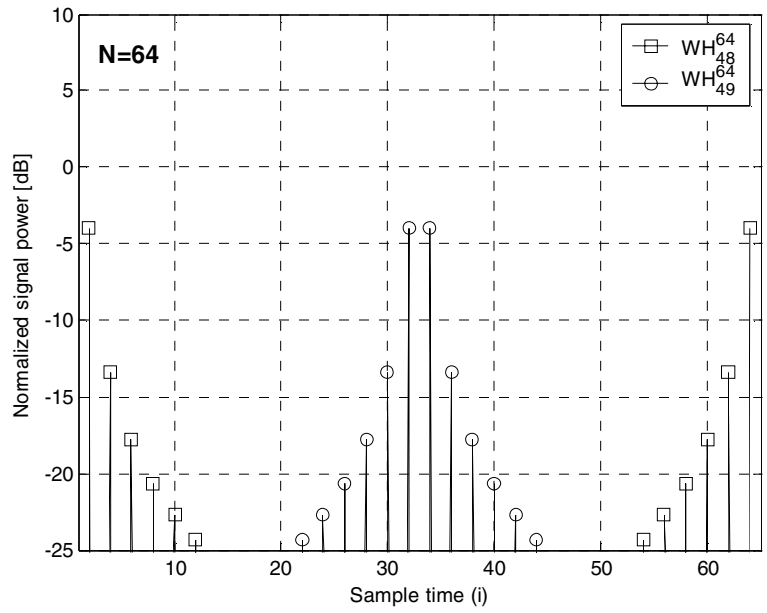

Fig. 6. Power of the proposed OFDM-CDMA signal with WH spreading code

when $N=64$.

The WH codes have a property that each pair of adjacent chips with an odd index and an even index have opposite signs and the same signs, respectively. For example, WH codes of length 4 can be represented as $W H_{0}^{4}=\{1,1,1,1\}$, $W H_{1}^{4}=\{1,-1,1,-1\}, W H_{2}^{4}=\{1,1,-1,-1\}, W H_{3}^{4}=\{1,-1,-1,1\}$, where $W_{k}^{l}$ denotes the $k$-th $\mathrm{WH}$ code with length $l$. Thus, WH spreading codes with an odd index have a preference for the allocation over those with an even index, since the WH code with an odd index make OFDM signal with $\cap$-shaped power characteristic and vice versa as depicts in Fig. 6. When a WH code is used as the spreading code, we propose a resource allocation rule for the OFDM-CDMA system given as

$$
m_{W H}(k)= \begin{cases}b_{(k-1) / 2}, & k=1,3, \ldots, N-1 \\ b_{(N+k) / 2}, & k=0,2, \ldots, N-2\end{cases}
$$

where $m_{W H}(k)$ denotes the modulation symbol allocated to the $k$-th WH spreading code of length $N$ (i.e., $W H_{k}^{N}$ ).

The OCI can further be reduced by employing a DFT basis as the spreading code. Let $D F T_{k}^{l}=e^{-j 2 \pi k n / l}$ be the $k$-th spreading code of length $l$ [13]. Since the inverse discrete Fourier transform (IDFT) of $D F T_{k}^{N}$ is an impulse function located at time $k$ as depicted in Fig. 7, the OFDM-CDMA signal using a DFT spreading code can be allocated as

$$
m_{D F T}(k)=b_{k}, k=0,1, \ldots, N-1
$$

where $m_{D F T}(k)$ denotes the modulation symbol allocated to the $k$-th DFT spreading code of length $N$. Thus, the power loss due to the $\mathrm{CP}$ can completely be eliminated when $K \leq\left(1-\eta^{-1}\right) N$ as depicted in Fig. 7, yielding substantial reduction of the OCI power.

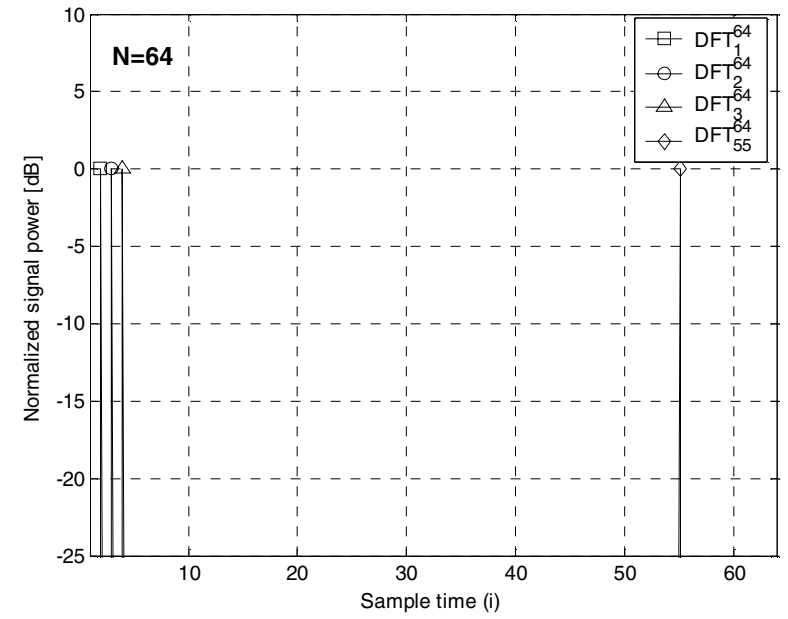

Fig. 7. Power of the proposed OFDM-CDMA signal with DFT code when

$N=64$.

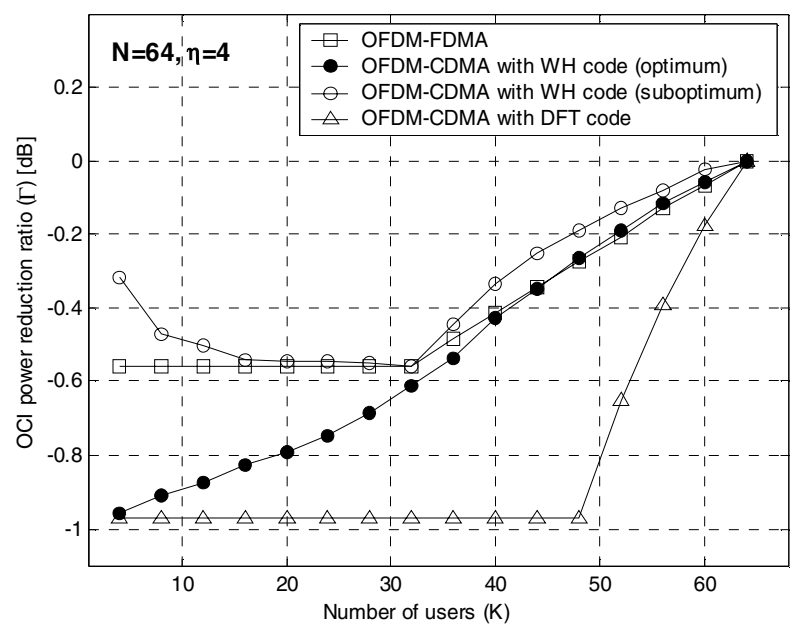

Fig. 8. The average OCI power reduction ratio as a function of $K$ when $N=64$ and $\eta=4$.

\section{PERFORMANCE EVALUATION}

The performance of the proposed resource allocation schemes is verified by computer simulation. When $N=64$ and $\eta=4$, Fig. 8 depicts the OCI power reduction ratio $\Gamma$ as a function of the number of users, $K$. It can be seen that the performance improvement is significant unless $K$ is too large. When applied to an OFDM-FDMA system, the proposed scheme reduces the OCI power by nearly up to $0.6 \mathrm{~dB}$ when $K \leq 32$. But, when $K>32, \Gamma$ increases as $K$ increases because $(K-32)$ pairs of symbols allocated to the adjacent subcarriers have the same signs. When applied to an OFDMCDMA with the use of WH codes, optimum code allocation 
rule provides OCI power reduction by nearly up to $1 \mathrm{~dB}$ when $K$ is very small. It can be seen that the OFDM-CDMA with the use of DFT spreading codes provides the performance better than the use of $\mathrm{WH}$ codes. The proposed scheme provides an OCI reduction of nearly $1 \mathrm{~dB}$ with the use of DFT code when $K \leq 48$ since $\beta$ is infinity (i.e., $\left.\Gamma_{\beta \rightarrow \infty}=\eta /(\eta+1)\right)$. When $K>48$, the resource will be allocated to the last portion of the OFDM symbol in the time domain, yielding performance degradation.

\section{CONCLUSIONS}

We have proposed resource allocation schemes that can reduce the OCI in OFDM-based asynchronous cellular systems by lowering the average power of the $\mathrm{CP}$, corresponding to the last portion of the OFDM symbol. The proposed resource schemes can easily be applied to OFDM-FDMA and OFDMCDMA with the use of orthogonal spreading codes. Simulation results show that the proposed schemes can reduce the OCI power by nearly up to $1 \mathrm{~dB}$ in typical cellular OFDM environment.

\section{APPENDIX}

\section{Power Characteristic of THE Proposed OFDM SignaL}

We prove that the proposed resource allocation method results in an OFDM signal with a $\cap$-shaped power characteristic. From the proposed resource allocation method (5), $X_{n}$ can be decomposed into two terms, $X_{n}^{e}$ and $X_{n}^{o}$, with odd and even indices as

$$
\begin{aligned}
& X_{n}^{e}= \begin{cases}b_{n / 2} / \sqrt{2}, & \text { even } n \\
0, & \text { odd } n\end{cases} \\
& X_{n}^{o}= \begin{cases}0, & \text { even } n \\
-b_{(n-1) / 2} / \sqrt{2}, & \text { odd } n .\end{cases}
\end{aligned}
$$

Then, the time domain signal can be obtained by the IDFT operation as

$$
\begin{aligned}
x_{i} & =\frac{1}{N} \sum_{n=0}^{N-1} X_{n} e^{j 2 \pi i n / N} \\
& =\frac{1}{N} \sum_{n=0}^{N-1}\left(X_{n}^{e}+X_{n}^{o}\right) e^{j 2 \pi i n / N}
\end{aligned}
$$

where $i$ is sample time index in OFDM symbol. Since $X_{n}^{o}=-X_{n-1}^{e}$, (A.2) can be rewritten as

$$
\begin{aligned}
x_{i} & =\frac{1}{N} \sum_{n=0}^{N-1}\left(X_{n}^{e}-X_{n-1}^{e}\right) e^{j 2 \pi i n / N} \\
& =\frac{1}{N}\left(1-e^{j 2 \pi i / N}\right) \sum_{n=0}^{N-1} X_{n}^{e} e^{j 2 \pi i n / N} .
\end{aligned}
$$

The average power at time $i$ can be obtained by

$$
\begin{aligned}
P_{x_{i}} & =E\left[\left|x_{i}\right|^{2}\right] \\
& =C S_{i}
\end{aligned}
$$

where

$$
\begin{gathered}
S_{i}=\left|1-e^{j 2 \pi i / N}\right|^{2} \\
C=\frac{1}{N^{2}} E\left[\left|\sum_{n=0}^{N-1} X_{n}^{e} e^{j 2 \pi i / N}\right|^{2}\right] .
\end{gathered}
$$

Note that $C$ is a constant indifferent from the time index $i$ in an average sense. Thus, the shape of $P_{x_{i}}$ depends only on that of $S_{i}$. Since $S_{i}$ has a $\cap$ shape, $P_{x_{i}}$ also has a $\cap$ shape. Note that $\beta$ can be obtained by

$$
\beta=\frac{\sum_{i=0}^{\left(1-\eta^{-1}\right) N-1} S_{i}}{(\eta-1) \sum_{i=\left(1-\eta^{-1}\right) N}^{N-1} S_{i}}
$$

where $\left(1-\eta^{-1}\right) N$ is an integer number corresponding to the number of samples during the OFDM symbol interval excluding the last portion corresponding to the CP.

\section{REFERENCES}

[1] R. V. Nee and R. Prasad, OFDM for wireless multimedia communications, Altech House, 2000

[2] J. A. C. Bingham, "Multicarrier modulation for data transmission: An idea whose time has come," IEEE Commun. Mag., vol. 28, no. 5, pp5-14, May 1990.

[3] S. Hara and R. Prasad, "Overview of multicarrier CDMA," IEEE Commun. Mag., vol. 35, no. 12, pp. 126-133, Dec. 1997.

[4] T. S. Rappaport, Wireless communications, Prentice Hall, first edition, 1996.

[5] S. Kaiser, "OFDM code-division multiplexing in fading channels," IEEE Trans. Commun., vol. 50, pp. 1266-1273, Aug. 2002.

[6] Flarion, The benefits of a packet-switched, ALL-IP mobile broadband network, Flarion white paper, Feb. 2004.

[7] J. C. Chuang and N. R. Sollenberger, "Dynamic packet assignment for advanced cellular internet service," in Proc. IEEE Globecom'97, pp. 1596-1600, Nov. 1997.

[8] S. Faruque, "High capacity cell planning based on fractional frequency reuse with optimum trunking efficiency," in proc. IEEE VTC'98, pp. 1458-1460, May 1998.

[9] 3GPP, Physical layer aspects for evolved universal terrestrial radio access (UTRA), TR 25.814 V7.0.0, June 2006

[10] A. Hutter, "Design of OFDM systems for frequency-selective and timevariant channels," in Proc. IEEE IZS'02, pp. 39-1-39-6, Feb. 2002.

[11] S. H. Tsai, Y. P. Lin and C.-C. Jay Kuo, "MAI-free MC-CDMA system based on Hardamard-Walsh codes," IEEE Trans. Signal Process., vol. 54, no. 8, pp. 3166-3179, Aug. 2006.

[12] Q. Shi and M. Latva-aho, "Simple spreading code allocation scheme for downlink MC-CDMA," Electron. Lett., vol. 38, no. 15, pp. 807-809, July 2002.

[13] G. Rath and C. Guillemot, "Performance analysis and recursive syndrome decoding of DFT codes for bursty erasure recovery," IEEE Trans. Signal Process., vol. 51, no. 5, pp. 1335-1350, May 2003. 